\title{
Diversity of bulbous monocots in Turkey with special reference. Chromosome numbers*
}

\author{
Neriman Özhatay ${ }^{\ddagger}$ \\ University of Istanbul, Faculty of Pharmacy, Department of Pharmaceutical Botany, \\ 34452 Ístanbul, Turkey
}

Abstract: Turkey is among the richest countries in the world in terms of plant diversity. The aim of this paper is to show diversity of the petaloid and bulbous plants, which belong to monocotyledones. According to Flora of Turkey and its supplements, the number of species of bulbous monocotyledones is around 688, of which 244 are endemic (endemism $35.4 \%$ ). According to the statistical data of Turkish flowering plants based on the published records, only $15 \%$ of the flora have information about chromosome counts, although bulbous monocots percentage is higher at $58 \%$. Fritillaria and Tulipa are stable genera as regards chromosome numbers with $2 \mathrm{n}=24$ and karyotype; on the other hand, the most variable genera are Crocus $(2 \mathrm{n}=6,8,10,12,14,16,18,20,22,26,28,30,70)$ and Ornithogalum $(2 \mathrm{n}=12$, $14,16,18,20,21,24,27,28,32,40,45,54,60,80)$.

\section{INTRODUCTION}

Turkey covers $779.452 \mathrm{~km}^{2}$, of which $3 \%$ lies in European Turkey (Thrace), the remainder in Asian Turkey (Anatolia). With around 9000 species, the flora is the richest of any country in Europe, North Africa, and the Middle East, and indeed is more typical of a continent or tropical country than an individual temperate country. Of these, nearly one in every three plants is endemic, an astonishingly high percentage for a mainland country (Table 1).

Table 1 Wild flowering plants species in Turkey [1-3].

\begin{tabular}{lccccc}
\hline Family & Genera & Species & $\begin{array}{c}\text { Total infrageneric taxa } \\
\text { (ssp. \& var.) }\end{array}$ & Endemic taxa & Endemism \\
\hline 142 & 1138 & 8930 & 10653 & 3707 & $34.5 \%$ \\
\hline
\end{tabular}

The main reasons for this wealth are as follows:

- $\quad$ a variety of climates

- $\quad$ topographical diversity with marked changes in ecological factors over a short distance

- geological and geomorphic variation

- a range of aquatic environments such as seas, lakes, and rivers

- $\quad$ altitude variations from sea level to $5000 \mathrm{~m}$

- There are a number of major mountain ranges in Anatolia that constitute effective barriers, and these have further encouraged a greater diversity of species

\footnotetext{
* Lecture presented at the $3^{\text {rd }}$ IUPAC International Conference on Biodiversity (ICOB-3), Antalya, Turkey, 3-8 November 2001. Other presentations are presented in this issue, pp. 511-584.

‡Corresponding author. E-mail: n.özhatay@ixir.com
} 
- The situation of the country at the junction of three major phytogeographical regions: the EuroSiberian element that predominates in the cooler northern parts of the country; the Mediterranean element that is mainly restricted to the mild climatic zone along Turkey's southern and western shores; and the Irano-Turanian element, characteristic of the vast steppes and mountains of Inner Anatolia with a continental climate characterized by soaring summer temperatures, and often bitterly cold winters.

- Additionally, during many historical periods, Anatolia has served as a passage way between the continents of Europe, Asia, and Africa, resulting in the dispersal of a wide variety of plants and animals.

All of these factors combined have provided many opportunities for the plants to evolve and differentiate, creating the present wealth of species now estimated to be over 8930, about one-third of which are endemics.

\section{BULBOUS PLANTS IN TURKEY}

It is also a well-known fact to botanists that Turkey is among the world's most richly endowed countries with regard to bulbous monocotyledones. It has the richest variety in the world, with greater numbers of Fritillaria and Crocus, for instance, than any other country. This wealth mirrors the general richness of Turkish flora. This diversity stems principally from Turkey's geographical position, sandwiched between the steppes of central Asia, the deserts of Syria, the cold forested mountains of Central Europe, and the Mediterranean to the west. Many of Turkey's varied climates are ideal for bulbs, being moist in the spring or autumn, when bulbs flower and seed, and dry in the summer, when the specially adapted bulbs are able to lie dormant during the extremes of heat and drought.

Table 2 Monocotyledones in Turkey.

\begin{tabular}{lccccc}
\hline Family & Genera & Species & $\begin{array}{c}\text { Total infrageneric taxa } \\
\text { (subsp. \& var.) }\end{array}$ & Endemic taxa & Endemism \\
\hline $24 \quad 251$ & 1450 & 1731 & 389 & $22.5 \%$ \\
\multicolumn{2}{l}{$\begin{array}{l}\text { Petaloid and bulbous } \\
\text { monocotyledones in Turkey }\end{array}$} & 688 & 244 & $35.4 \%$ \\
\hline
\end{tabular}

\section{THREATS OF THE TURKISH BULBS}

Commercial collection and trade represents the principle threat to individual species. Among the groups of plants most affected are medicinal plants, orchids, and bulbous plants. Özhatay et al. [4] have documented the commercial trade in medicinal plants, identifying some 346 taxa either in internal commerce and exported. On average, Turkey exports 28.000 tons of medicinal and aromatic plants annually, generating approximately USD 50 million in foreign income. A number of conservationists regard the trade in orchid tubers to be the most worrying. Annually, it is estimated that up to 30 million tubers, of some 40 species, are collected, to be dried and powdered for the production of a warm winter drink ("salep") and a distinctive ice cream ("Maras Dondurması").

The best-documented example of the trade in a group of plants is that of the bulbous plants collected for the horticultural trade. Since the 1960s, the collection of wild bulbs has increased annually, reaching a peak of over 70 million tubers in late 1980s. Conservationists feared that for at least some species (e.g., Cyclamen spp., Galanthus elwesii, G. woronowii, Fritillaria imperialis, F. persica, etc.), such levels of collection were unsustainable. Rather than simply seeking a ban within the importing 
countries, conservationists, and Turkish scientists have collectively promoted programs to artificially propagate and cultivate bulbs in village locations, thereby reducing pressures on wild populations, yet at the same time maintaining valuable income to village communities. While the switch from collection to cultivation is taking longer than previously expected, the signs are encouraging. Today, a large quantity of Leucojum aestivum, Fritillaria imperialis, and F. persica is produced from cultivated sources.

However, the greater threat to a wider range of Turkish plants arises indirectly, through damage and destruction of their habitats.

\section{CHROMOSOME NUMBERS OF TURKISH PETALOID AND BULBOUS MONOCOTS}

The Flora of Turkey and the East Aegean Islands [1-3] includes 10653 flowering plant taxa, only 1613 of them have chromosome numbers [5]. All of the counts are taken from published papers, and all chromosome counts have been traced in the available literature (only material of known wild Turkish origin, which has been cultivated in Turkey or cultivated elsewhere from material of wild Turkish origin).

According to the statistical data of Turkish flowering plants based on the supplements Vols. 10 and 11 of the Flora of Turkey [2,3], only chromosome numbers of 1613 taxa are counted (Fig. 1).

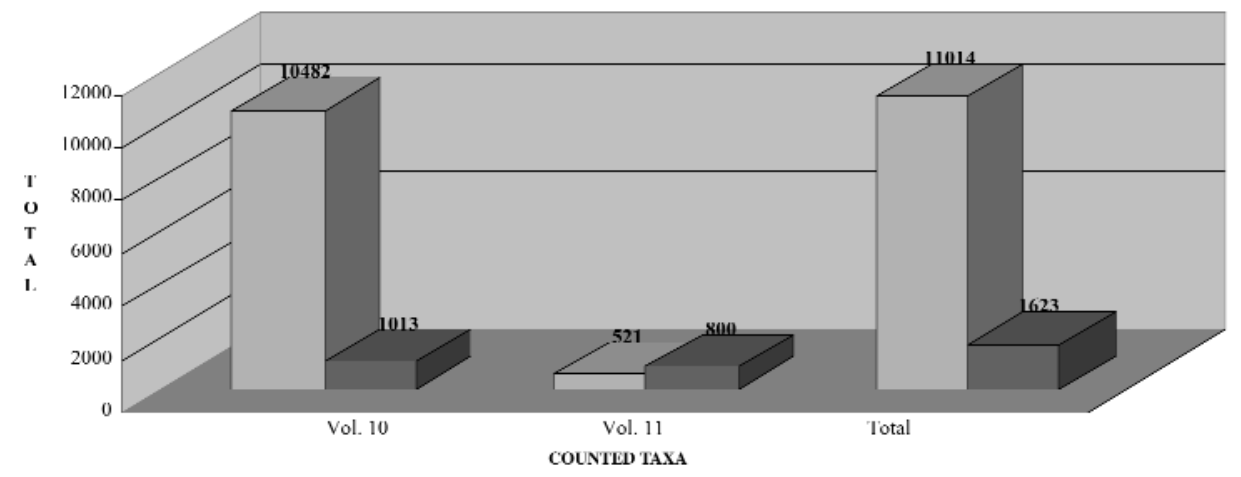

Fig. 1 Chromosome numbers of counted taxa of Turkish flowering plants.

In spite of the floristic richness of Turkey, only $15 \%$ of the flora has information about the chromosome counts. In comparison, counted chromosome numbers of petaloid and bulbous monocots are considerably higher than the rest of the Turkish flowering plant taxa (Fig. 2).
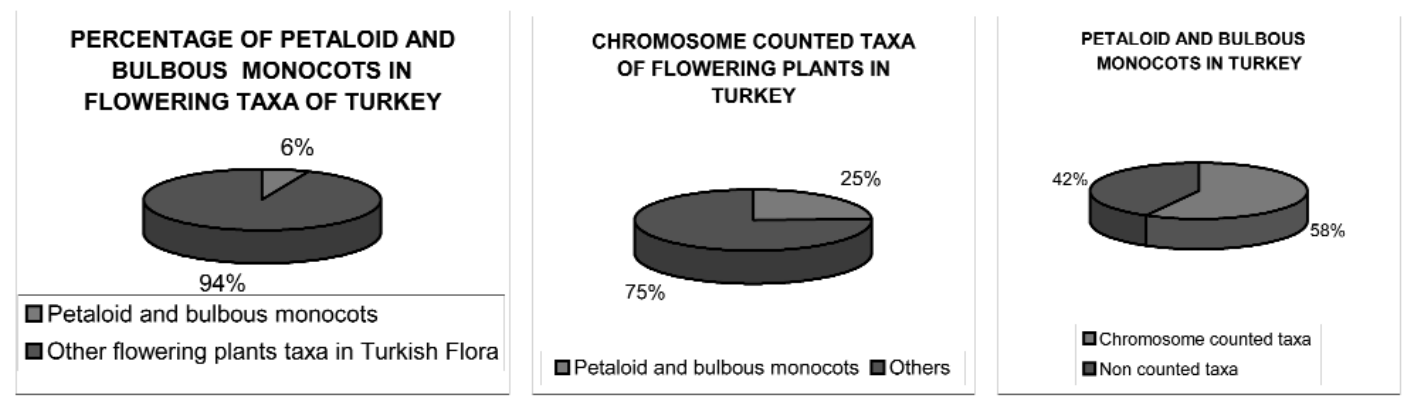

Fig. 2 Percentage of chromosome numbers of counted taxa of petaloid and bulbous monocots in Turkey. 
The list of the petaloid and bulbous monocotyledones in Turkey with the chromosome number are given below (Table 3).

Table 3 List of the chromosome numbers of Turkish petaloid and bulbous monocots.

\begin{tabular}{|c|c|c|c|}
\hline Taxon & Chromosome number (2n) & Taxon & Chromosome number $(2 \mathrm{n})$ \\
\hline \multicolumn{4}{|l|}{ Amaryllidaceae } \\
\hline Galanthus elwesii & 24 & C. biflorus ssp. isauricus & $8,10,12$ \\
\hline G. fosteri & 24 & C. biflorus ssp. nubigena & 12 \\
\hline G. gracilis & 24 & C. biflorus ssp. pseudonubigena & 18,20 \\
\hline G. nivalis ssp. cilicicus & 24 & C. biflorus ssp. pulchricolor & 8 \\
\hline G. nivalis ssp. nivalis & 24 & C. biflorus ssp. punctatus & 8 \\
\hline G. peshmenii & 24 & C. biflorus ssp. tauri & 20,22 \\
\hline G. rizehensis & 24 & C. cancellatus ssp. cancellatus & 10 \\
\hline Leucojum aestivum & 22,24 & C. cancellatus ssp. damascenus & $8,10,10+4 B, 12$ \\
\hline Sternbergia candida & 20 & C. cancellatus ssp. lycius & 14 \\
\hline S. clusiana & 20 & C. cancellatus ssp. mazziaricus & 16 \\
\hline S. colchiciflora & 20,10 & C. cancellatus ssp. pamphlicus & 12 \\
\hline S. fischeriana & 20 & C. candidus & 6 \\
\hline S. lutea & 22,33 & C. chrysanthus & $8,10,12,14,16$ \\
\hline S. sicula & 24 & C. danfordiae & 8 \\
\hline Araceae & & C. flavus ssp. dissectus & 8 \\
\hline Arum byzantinum & 28 & C. flavus ssp. flavus & $8,8+11 \mathrm{~B}$ \\
\hline A. creticum & 28 & C. fleischeri & 20 \\
\hline A. dioscorides & 28 & C. gargaricus & 30 \\
\hline A. elongatum & 28 & C. graveolens & 6 \\
\hline A. euxinum & 28 & C. karduchorum & 10 \\
\hline A. italicum & 70,84 & C. kotschyanus s.l. & 20 \\
\hline A. maculatum & 56 & C. kotschyanus ssp. cappadocicus & 10 \\
\hline A. nickelii & 84 & C. kotschyanus ssp. hakkariensis & 10 \\
\hline A. orientale & 28 & C. kotschyanus ssp. kotschyanus & 8,10 \\
\hline $\begin{array}{l}\text { Biarum davisii ssp. } \\
\text { marmarisense }\end{array}$ & 26 & $\begin{array}{l}\text { C. kotschyanus ssp. } \\
\text { suworowianus }\end{array}$ & 20 \\
\hline B. ditschianum & 26 & C. laevigatus & 26 \\
\hline B. eximium & 16 & C. leichtlinii & 20 \\
\hline B. kotschyi c. & 96 & C. mathewii & 70 \\
\hline Eminium koenenianum & 28 & C. olivieri ssp. balansae & 6 \\
\hline Iridaceae & & C. pallasii ssp. dispathaceus & 14 \\
\hline Crocus abantensis & 16 & C. pallasii ssp. pallasii & 14 \\
\hline C. adanensis & 14 & C. pallasii ssp. turcicus & 12 \\
\hline C. aerius & 22,26 & C. pestalozzae & 28 \\
\hline C. ancyrensis & 10 & C. pulchellus & 12 \\
\hline C. antalyensis & 8 & C. reticulatus ssp. hititicus & 10 \\
\hline C. asumaniae & 26 & C. reticulatus ssp. reticulatus & 10 \\
\hline C. baytopiorum & 28 & C. sativus & 24 \\
\hline C. biflorus s.l. & 8,10 & C. schorojanii & 8 \\
\hline C. biflorus ssp. biflorus & 8,10 & C. sieheanus & 16 \\
\hline C. biflorus ssp. crewei & 10 & C. speciosus $\mathrm{ssp}$. lgazensis & 6,8 \\
\hline C. speciosus ssp. speciosus & $8,10,18$ & A. charaulicum & 16 \\
\hline C. speciosus ssp. xantholaimos & 10 & A. chrysantherum & $16+1$ \\
\hline C. tournefortii & 30 & A. commutatum & 32 \\
\hline C. vallicola & 8 & A. coppoleri & 32 \\
\hline C. vitellianus & 8 & A. cupani & 16 \\
\hline Gladiolus anatolicus & 60 & A. cupani ssp. hirtovaginatum & 14 \\
\hline G. antakiensis & 120 & A. curtum & $16,16+4 B$ \\
\hline G. atroviolaceus & 80 & A. cyrilli & 16,32 \\
\hline
\end{tabular}

(continues on next page) 
Table 3 (Continued)

\begin{tabular}{|c|c|c|c|}
\hline Taxon & Chromosome number (2n) & Taxon & Chromosome number $(2 \mathrm{n})$ \\
\hline G. illyricus & 60 & A. deciduum ssp. deciduum & 16 \\
\hline G. kotschyanus & 60 & A. dentiferum & 32 \\
\hline Iris albicans & 44 & A. dictyoprasum & 16 \\
\hline I altica & 16 & A. djimilense & 16 \\
\hline I. danfordiae & 18 & A. eldivanense & 16 \\
\hline I. germanica & 44 & A. enginii & 16 \\
\hline I. histrio & 20 & A. fethiyense & 16 \\
\hline I. histrioides & 16 & $\begin{array}{l}\text { A.. flavum ssp. } \\
\text { flavum var. flavum }\end{array}$ & 16,32 \\
\hline I. junonia & 48 & A. flavum ssp. tauricum & 16,32 \\
\hline I. lazica & 32 & $\begin{array}{l}\text { A. flavum ssp. } \\
\text { tauricum var. tauricum }\end{array}$ & 16 \\
\hline I. pamphylica & 20 & A. fulcoviolaceum & $16,16+1 B$ \\
\hline I. persica & 24 & A. glumaceum & 16 \\
\hline I. pseudoacorus & 34 & A. gramineum & 16 \\
\hline I. purpureobractea & $24,48,49,72$ & A. guttatum ssp. dalmaticum & 16 \\
\hline I. reticulata & 18,20 & A. guttatum ssp. guttatum & $16,32,40$ \\
\hline I. schachtii & 48 & A. guttatum ssp. sardoum & 16 \\
\hline I. sintenisii & 16 & A. hirtovaginatum & 16 \\
\hline I. suaveolens & 24 & A. llgazense & 16 \\
\hline I. taochia & 24 & A. incisum & 16 \\
\hline Liliaceae & & A. jubatum & 16 \\
\hline Allium affine & 16 & A. junceum ssp. junceum & 16 \\
\hline A. akaka & 16 & A. junceum ssp. tridentatum & 16 \\
\hline A. amethystinum & 16 & A. karamanoglui & 16 \\
\hline A. ampeloprasum & $24,32,40,48$ & A. karsianum & 16 \\
\hline A. amphipulchellum & 16 & A. karyeteini & 16 \\
\hline A. anatolicum & 16 & A. kastambulense & 16 \\
\hline A. armenum & 16 & A. kharputense & 16 \\
\hline A. artvinense & 16 & A. kossoricum & 16 \\
\hline A. asperiflorum & 16 & A. kunthianum & 16 \\
\hline A. atropurpureum & 16 & A. lacerum & 16 \\
\hline A. atroviolaceum & $16,24,32$ & A. lycaonicum & 16,24 \\
\hline A. aucheri & 16 & A. machrochaetum & 16 \\
\hline A. bassilense & 16 & A. moschatum & 16 \\
\hline A. baytopiorum & 16 & A. myrianthum & 16 \\
\hline A. bourgeaui ssp. bourgeaui & 16 & A. neapolitanum & $21,28,35,42$ \\
\hline A. bourgeaui ssp. cycladicum & 16 & A. nevsehirense & 16,32 \\
\hline $\begin{array}{l}\text { A. callimischon ssp. } \\
\text { haemostictum }\end{array}$ & 16 & A. nigrum & 16 \\
\hline A. cappadocicum & $16,16+1 B$ & A. oltense & 16 \\
\hline A.cardiostemon & 16 & A. olympicum & 16 \\
\hline A. oreophilum & 16 & Bellevalia anatolica & 8 \\
\hline A. orientale & 16 & B. clusiana & 8 \\
\hline A. pallens ssp. pallens & 16 & B. dubia & 8 \\
\hline A. paniculatum ssp. fuscum & 16,24 & B. edirnensis & 24 \\
\hline $\begin{array}{l}\text { A. paniculatum ssp. } \\
\text { paniculatum }\end{array}$ & $16,32,40$ & B. fominii & 8 \\
\hline A. paniculatum ssp. villosulum & 16 & B. forniculata & 8 \\
\hline A. peroninianum & 16 & B. gracilis & $8+3 B, 16$ \\
\hline A. phrygium & 16 & B. kurdistanica & 16 \\
\hline A. ponticum & 32 & B. latifolia & 32 \\
\hline A. proponticum & 16 & B. longistyla & $31,32,32+\mathrm{B}, 35$ \\
\hline A. pseudoalbidum & 16 & B. mathewii & 8 \\
\hline
\end{tabular}

(continues on next page) 
Table 3 (Continued)

\begin{tabular}{|c|c|c|c|}
\hline Taxon & Chromosome number (2n) & Taxon & Chromosome number $(2 n)$ \\
\hline A. pseudampeloprasum & 16 & B. modesta & 8 \\
\hline A. pseudflavum & 16 & B. paradoxa & $8,8+3 \mathrm{~B}$ \\
\hline A. reuterianum & 16 & B. pycnantha & $16,16+2 B$ \\
\hline A. rhodopeum ssp. tauricum & 16 & B. rixii & 8 \\
\hline A. rollovii & 16 & B. sarmatica & $8,8+2 B, 8+3 B$ \\
\hline A. roseum & 40 & B. tauri & 16 \\
\hline A. rotundum s.l & 32 & B. trifoliata & 8 \\
\hline A. rotundum ssp. jajlae & 16,32 & Colchicum baytopiorum & $46,54,108$ \\
\hline A. rotundum ssp. rotundum & $16,16+2 B, 32,40$ & C. bivonae & 36 \\
\hline A. rotundum ssp. waldsteinii & 24 & C. boissieri & 46 \\
\hline A. rupestre & 16 & $\begin{array}{l}\text { C. chalcedonicum ssp. } \\
\text { chalcedonicum }\end{array}$ & 54 \\
\hline A. rupicola & $16+B$ & $\begin{array}{l}\text { C. chalcedonicum ssp. } \\
\text { punctatum }\end{array}$ & 50 \\
\hline A. sandrasicum & 16 & C. cilicicum & 54 \\
\hline A. scabriflorum & 16 & C. davisii & 46 \\
\hline A. schoenoprasum & 16 & C. decaisnei & 54 \\
\hline A. scorodoprasum & 16,32 & C. dolichantherum & 54 \\
\hline A. sieheneanum & $24+1 B$ & C. heldreichii & 54 \\
\hline A. sipyleum & 16 & C. imperatoris-friderici & 54 \\
\hline A. sivasicum & 16 & C. inundatum & 54 \\
\hline A. sosnowskyanum & 16 & C. kotschyi & 20 \\
\hline A. sphaerocephalon s.l. & 16,32 & C. lingulatum s.l. & 54 \\
\hline $\begin{array}{l}\text { A. sphaerocephalon ssp. } \\
\text { sphaerocephalon }\end{array}$ & 16 & C. lingulatum ssp. rigescens & 54 \\
\hline A. stamineum & 16 & C. macrophyllum & 54 \\
\hline $\begin{array}{l}\text { A. stearnianum ssp. } \\
\text { stearnianum }\end{array}$ & 16 & C. micaceum & 54 \\
\hline A. stenopetalum & 16 & C. minutum & 44 \\
\hline A. stylosum & 16 & C. munzurense & 24 \\
\hline A. subhirsutum & 14 & C. paschei & 48 \\
\hline A. szovitsii & 16,56 & C. persicum & 54 \\
\hline A. tchihatschewii & 16 & C. sanguicolle & 22 \\
\hline A. trachycoleum & 32,48 & C. speciosum & 38 \\
\hline A. tuncelianum & 16 & C. triphyllum & 42 \\
\hline A. turcicum & 16,17 & C. turcicum & 52 \\
\hline A. vineale & 32 & C. variegatum & 44 \\
\hline A. wendelboanum & 16 & Fritillaria bithynica & 24 \\
\hline A. wiedemannianum & 16 & F. carica & 24 \\
\hline A. zebdanense & 18 & F. crassifolia ssp. hakkariensis & 24 \\
\hline F. elwesii & 24 & O. arcuatum & 26,32 \\
\hline F. imperialis & 36 & O. armeniacum & $\begin{array}{c}14,16,18,18+1 \mathrm{~B}, 20 \\
20+1 \mathrm{~B}, 20+4 \mathrm{~B}\end{array}$ \\
\hline F. minima & 24 & O. arabicum & 28 \\
\hline F. minuta & 24 & O. balansae & $12,24,24+1 \mathrm{~B}$ \\
\hline F. persica & 24 & O. comosum & $14,16,18,20$ \\
\hline F. pontica & 24 & O. euxinum & 18 \\
\hline F. sororum & 24 & O. fimbriatum & $12,13,35,36,20+1 \mathrm{~B}$ \\
\hline F. stribrnyi & 24 & O. gussonei & 14,18 \\
\hline Gagea bohemica & 36 & O. improbum & 18 \\
\hline G. chrysantha & 36 & O. joschtiae & 18 \\
\hline G. pratensis & 36 & o. kuereanum & 14 \\
\hline G. villosa var. villosa & $24,36,48$ & O. lanceolatum & $18,20,22$ \\
\hline Hyacinthella acutiloba & 18 & O. luschanii & $22,28,44,60,76$ \\
\hline
\end{tabular}

(continues on next page) 
Table 3 (Continued)

\begin{tabular}{|c|c|c|c|}
\hline Taxon & Chromosome number $(2 \mathrm{n})$ & Taxon & Chromosome number $(2 n)$ \\
\hline H. campanulata & 18 & O. macrum & 54 \\
\hline H. glabracens & 18,20 & O. montanum & 14,18 \\
\hline H. heldreichii & 18 & O. mysum & 16 \\
\hline H. hispida & 18 & O. narbonense & $\begin{array}{l}14,16,18,22,24,28 \\
14+1 \mathrm{~B}, 2 \mathrm{~B}, 5 \mathrm{~B}, 36,46\end{array}$ \\
\hline H. lazulina & 22 & O. nivale & 14 \\
\hline H. lineata & 16 & O. nutans & $14,15,18,40$ \\
\hline H. nervosa & 24 & O. oligophyllum & $\begin{array}{l}16,18,20,24,24+2 \\
\quad 40,80\end{array}$ \\
\hline H. siirtensis & 22 & O. orthophyllum & $14,16,28$ \\
\hline H. venusta & 22 & O. pascheanum & 14 \\
\hline $\begin{array}{l}\text { Hyacinthus orientalis ssp. } \\
\text { orientalis }\end{array}$ & 16 & O. platyphyllum & $16,18,27,54$ \\
\hline $\begin{array}{l}\text { H. orientalis subsp. } \\
\text { chionophilus }\end{array}$ & 16 & O. pluttulum & 14 \\
\hline Merendera trigyna & 22 & O. pyrenaicum & $16,18,24$ \\
\hline Muscari anatolicum & 18 & O. reflexum & 12 \\
\hline M. armeniacum & 18 & O. refractum & 28,54 \\
\hline M. aucheri & $18,36,37$ & O. saginatum & 28 \\
\hline M. azureum & 18 & O. shelkovnikovii & 26 \\
\hline M. bourgaei & 18 & O. sibthorpii & $14,28,32$ \\
\hline M. caucasicum & 18 & O. sigmoideum & $\begin{array}{l}12,14,16,16+B, 19,20 \\
24,34\end{array}$ \\
\hline M. commutatum & 18,27 & O. sorgerae & 18 \\
\hline M. coelesta & 18 & O. sphaerocarpum & 16 \\
\hline M. comosum & 18 & O. ulophyllum & $14,16,18$ \\
\hline M. discolor & 18 & O. uluense & 20 \\
\hline M. latifolium & $18,18+1 B$ & O. umbellatum & $16,36,44,45,54$ \\
\hline M. longipes & 18 & O. wiedemannii & $\begin{array}{c}12,12+1 \mathrm{~B}, 14,14+1 \\
14+2 \mathrm{~B}, 18,21,22\end{array}$ \\
\hline M. macrocarpum & 18 & Scilla autumnalis & $14,16,28,41$ \\
\hline M. massayanum & 18 & S. bifolia & $14,18,36$ \\
\hline M. microstomum & 18 & S. bithynica & 12 \\
\hline M. mirum & 18 & S. cilicica & 12 \\
\hline M. muscarimi & 18 & S. dedea & 18 \\
\hline M. neglectum & $18,28,36,36+1,45,54$ & S. forbesii & 18 \\
\hline M. sandrasicum & 18 & S. hyacinthoides & 20 \\
\hline M. tenuiflorum & $18,18+1 B$ & S. ingridae & 12 \\
\hline M. weissii & 18 & S. leepii & 12 \\
\hline Ornithogalum alpigenum & 18 & S. melaina & 12 \\
\hline O. aemulum & 16 & S. monanthos & 12 \\
\hline S. persica & 8 & T. thracica & 48 \\
\hline S. pruinosa & 36 & T. undulatifolia & 36 \\
\hline S. rosenii & 12 & $\begin{array}{l}\text { Nectaroscordum siculum ssp. } \\
\text { siculum }\end{array}$ & 18 \\
\hline S. siberica ssp. armena & 12 & N. siculum subsp. bulgaricum & 18 \\
\hline Tulipa armena & 24 & Urginea maritima & $20,40,50$ \\
\hline T. biflora & 24 & Orchidaceae & \\
\hline T. cinnabarina & 24 & Orchis coriophora & 38 \\
\hline T. hellespontica & 48 & O. morio ssp. morio & 36 \\
\hline T. orphanidea & 36 & O. purpurea & 42 \\
\hline T. sylvestris & 24 & O. tridentata & 42 \\
\hline T. sylvestris $\mathrm{ssp}$. australis & 24,36 & & \\
\hline
\end{tabular}




\section{RESULTS AND DISCUSSION}

All of the counts of this presentation are taken from published papers. The Flora of Turkey and the East Aegean Islands [1-3] include 11014 flowering plant taxa, of which 1623 chromosome numbers have been recorded. The chromosome numbers of 366 taxa of Turkish petaloid and bulbous monocotyledones have been counted. Table 4 summarizes genera of which chromosomes are counted with the number of species and endemic taxa.

Table 4 Genera of petaloid and bulbous monocots with their species numbers, endemism, and chromosome numbers.

\begin{tabular}{|c|c|c|c|c|c|}
\hline Genus & $\begin{array}{l}\text { Species+ } \\
\text { subsp./var. }\end{array}$ & $\begin{array}{l}\text { Endemic } \\
\quad \text { taxa }\end{array}$ & $\begin{array}{l}\text { Endemism } \\
\qquad \%)\end{array}$ & $\begin{array}{l}\text { Chromosome } \\
\text { counted } \\
\text { taxa }\end{array}$ & Chromosome numbers $(2 \mathrm{n})$ \\
\hline Allium & $164+41$ & 65 & $40 \%$ & 111 & $\begin{array}{l}14,16,18,21,24,28,32,35,40,42, \\
48,56\end{array}$ \\
\hline Arum & $11+8$ & 2 & $18 \%$ & 9 & $25,56,70,84$ \\
\hline Bellevalia & 21 & 10 & $43 \%$ & 18 & $8,16,24,31,32,35$ \\
\hline Biarum & $7+2$ & 2 & $29 \%$ & 4 & $16,26,96$ \\
\hline Chionodoxa & 3 & 3 & $100 \%$ & 3 & 18,27 \\
\hline Colchicum & 35 & 14 & $40 \%$ & 26 & $\begin{array}{l}20,22,24,36,38,42,44,46,50,52, \\
54,108\end{array}$ \\
\hline Crocus & $36+34$ & 22 & $61 \%$ & 54 & $\begin{array}{l}6,8,10,12,14,16,18,20,22,26 \\
28,30,70\end{array}$ \\
\hline Eminium & $3+3$ & 1 & $33 \%$ & 1 & 28 \\
\hline Fritillaria & $36+12$ & 19 & $53 \%$ & 11 & 24 \\
\hline Gagea & $25+2$ & 2 & $8 \%$ & 4 & $24,36,48$ \\
\hline Galanthus & $13+4$ & 2 & $14 \%$ & 7 & 24 \\
\hline Gladiolus & 9 & 4 & $44 \%$ & 5 & 10 \\
\hline Iris & 39 & 14 & $36 \%$ & 17 & $16,18,20,24,32,44,48,49,72$ \\
\hline Hyacinthella & 11 & 10 & $91 \%$ & 10 & $16,18,20,22,24$ \\
\hline Hyacinthus & $1+2$ & 1 & $50 \%$ & 2 & 16 \\
\hline Lеисојит & 1 & - & - & 1 & 22,24 \\
\hline Merendera & 4 & - & - & 1 & 22 \\
\hline Muscari & 26 & 16 & $62 \%$ & 21 & $18,27,28,36,37,45,54$ \\
\hline Nectaroscordum & $2+2$ & - & - & 2 & 18 \\
\hline Orchis & $22+6$ & - & - & 4 & $36,38,42$ \\
\hline Ornithogalum & $37+2$ & 14 & $38 \%$ & 39 & $\begin{array}{l}12,13,14,15,16,18,19,20,21,22, \\
24,26,27,28,32,40,44,45,54, \\
60,76,80\end{array}$ \\
\hline Puschkinia & 1 & - & - & 1 & 10 \\
\hline Scilla & 16 & 4 & $25 \%$ & 15 & $8,12,14,16,18,20,28,36,41$ \\
\hline Sternbergia & 7 & 2 & $29 \%$ & 6 & $10,20,22$ \\
\hline Tulipa & 15 & 3 & 20 & 9 & $24,36,48$ \\
\hline Urginea & 1 & - & - & 1 & $20,20,50$ \\
\hline
\end{tabular}

Chromosome numbers of whole taxa of five genera Hyacinthus, Chionodoxa, Leucojum, Puschkinia, and Urginea are counted. On the other hand, none of species has information on any of the genera Lilium, Arisarum, Ixiolirion, and many Orchidaceae. 


\section{REFERENCES}

1. P. H. Davis (Ed.). Flora of Turkey and the East Aegean Islands, Vols. 1-9, University Press, Edinburgh (1965-1985).

2. P. H. Davis (Ed.). Flora of Turkey and the East Aegean Islands, Supp. I, University Press, Edinburgh (1988).

3. A. Güner, N. Özhatay, T. Ekim, K. H. C. Başer. Flora of Turkey and the East Aegean Islands, Second Supplement, Vol. 11, p. 656, Edinburgh (2001).

4. N. Özhatay, M. Koyuncu, S. Atay, A. Byfield. Türkiye’nin Doğal Tıbbi Bitkilerinin Ticareti Hakkında Bir Çalışma (The Wild Medicinal Plant Trade in Turkey), Doğal Hayatı Koruma Derneği, Ístanbul, Türkiye (1997).

5. N. Sadıkoğlu and N. Özhatay. "Evaluation of Chromosome Counts of Flora of Turkey" in Proceedings of the $2^{\text {nd }}$ Balkan Botanical Congress (14-18 May 2000, Ístanbul), N. Özhatay (Ed.), 1, 331-336 (2001). 